\title{
Feasibility of Providing Unguided Online Cognitive Behavioural Therapy for Insomnia to Community- Dwelling Older Adults in Western Australia: A Non- Randomised Feasibility Study
}

\section{Yvonne Kutzer ( $\nabla$ y.kutzer@ecu.edu.au )}

Edith Cowan University - Joondalup Campus: Edith Cowan University https://orcid.org/0000-00033568-5030

\section{Lisa Whitehead}

Edith Cowan University - Joondalup Campus: Edith Cowan University

\section{Eimear Quigley}

Edith Cowan University - Joondalup Campus: Edith Cowan University

\section{Mandy Stanley}

Edith Cowan University - Joondalup Campus: Edith Cowan University

\section{Research}

Keywords: Older adults, insomnia, cognitive therapy, digital literacy, cognitive behavioral therapy for insomnia (CBT-I), online psychological intervention

Posted Date: June 16th, 2021

DOI: https://doi.org/10.21203/rs.3.rs-604623/v1

License: (c) (i) This work is licensed under a Creative Commons Attribution 4.0 International License.

Read Full License 


\section{Abstract}

Background: Approximately $60 \%$ of adults over the age of 60 years report that they have trouble sleeping. Online Cognitive Behavioural Therapy for insomnia (CBT-I), an evidence-based form of talk therapy, is considered an effective treatment option. This feasibility study examined the suitability of an online CBT-I programme for older adults aged $60-80$ years in Western Australia.

Methods: Participants with and without insomnia completed the first out of four online CBT-I modules and provided feedback via an online questionnaire regarding programme acceptability, utility and accessibility.

Results: High approval ratings (91.7\%) suggest that study participants believed the online CBT-I programme to be acceptable, useful and accessible, independent of their insomnia status. Potential areas for improvement were noted, including making the online programme more customisable and interactive.

Conclusions: Findings from this feasibility study indicate that respondents viewed the online CBT-I programme as appropriate for adults aged 60-80 years. The full-scale study will subsequently be conducted without any protocol alterations.

\section{Key Messages Regarding Feasibility}

1) What uncertainties existed regarding the feasibility?

It was unknown whether adults aged 60-80 years would be open to engaging with an online, unsupported Cognitive Behavioural Therapy (CBT) programme for insomnia, due tounknown digital literacy levels within this age group in Western Australia.

2) What are the key feasibility findings?

Findings from this feasibility study indicated that respondents viewed the programme as appropriate for adults aged 60-80 years. The main area of improvement for future research is to make the intervention more tailored to individual circumstances.

3) What are the implications of the feasibility findings for the design of the main study?

The outcomes of the feasibility study indicate an appropriate level of digital literacy and engagement in older adults in Western Australia. As a result, the full-scale study can commence without any significant alterations of the intervention protocol.

\section{Background}

Older adult populations report a high rate of sleep complaints, with almost $60 \%$ of adults over the age of 60 years describing at least one chronic sleep problem. ${ }^{1,2}$ Insomnia is characterised by difficulties with 
sleep initiation, sleep maintenance or early awakening, combined with a reduction in daytime functioning, and is considered chronic if it persists for at least three months, occurring a minimum of three nights a week. ${ }^{3}$ Furthermore, for a diagnosis of insomnia to be made, both poor sleep quality and a sleep complaint must be present. It is often assumed that poor sleep quality and sleep complaint occur simultaneously, however, this is not always the case. ${ }^{4}$ A subjective sleep complaint without objective poor sleep quality is termed paradoxical insomnia, ${ }^{5}$ and people with paradoxical insomnia are likely to also volunteer for research into sleep or see their GP about their sleep problems. However, on the other hand, some individuals may experience poor sleep but do not consider themselves to be experiencing insomnia. Those individuals with objective poor sleep in the absence of a sleep complaint, so called "noncomplaining poor sleepers", possibly have the same low levels of daytime impairment and anxiety as those who sleep well. Conversely, it has been proposed that those with a subjective sleep complaint but without objective poor sleep quality ("complaining good sleepers") may have worse health outcomes. It is estimated that about a quarter of the general population experience such uncoupling of sleep quality and complaint, although exact numbers are not known. ${ }^{4}$

Complaining good sleepers may display increased maladaptive or dysfunctional cognitions (e.g. "I didn't sleep all night so I will not be able to function today"), even though they do not report any functional effects, such as daytime sleepiness. Several studies have suggested that such dysfunctional beliefs might be important factors in the development and maintenance of insomnia, ${ }^{6-12}$ particularly in older adults. ${ }^{13}$ Cognitive behavioural therapy for insomnia (CBT-I) is generally the recommended first line treatment approach for those experiencing sleep problems. ${ }^{14}$ It can be delivered face-to-face in individual or group sessions or can be provided online (online CBT-I). ${ }^{15}$

CBT-I is thought to be effective at improving sleep quality in part because it addresses maladaptive beliefs and sleep-related distress, however, it is not known whether individuals with true insomnia respond differently to CBT-I than uncoupled sleepers (complaining good sleepers and non-complaining poor sleepers). A CBT-I programme can contain all, or some, of the following components: psychoeducation (e.g. sleep hygiene), cognitive components (cognitive therapy), and behavioural components of sleep restriction, stimulus control, and relaxation training. ${ }^{16}$ Psychoeducation about sleep hygiene focusses on creating the optimal sleep environment, such as appropriate bedroom temperature and light and noise reduction. In the cognitive therapy component the individual is taught to challenge unhelpful thoughts about sleep or awakening. The behavioural element of sleep restriction instructs the individual to adhere to a strict sleep - wake schedule to decrease time spent in bed awake, and also discourages day-time napping. This increases the homeostatic sleep drive, eventually leading to better nocturnal sleep. The stimulus control module addresses associations between the bedroom environment and sleep; this is achieved by encouraging the person with insomnia to get up and engage in other, preferably nonstimulating, activities if they cannot fall asleep within 15 minutes of going to bed. Finally, the relaxation training aims 
to decrease anxiety around sleep and reduces cognitive as well as physiological arousal. Techniques used include progressive muscle relaxation or deep breathing. Accessibility of CBT-I remains one of its main problems, due to the limited availability of trained professionals and the cost associated with delivering face-to-face CBT-I. ${ }^{17}$ The delivery of CBT-I as an online programme has been heralded as an alternative option to address these difficulties. In a systematic review and meta-analysis on online CBT-I programmes, Zachariae et $\mathrm{al}^{17}$ examined the usefulness of online CBT-I, its long-term outcomes and possible outcome moderators. They reported statistically significant results including a large effect size post-treatment for insomnia severity, medium effect sizes for sleep efficiency, and small effect sizes for sleep onset latency, wake after sleep onset, total sleep time, number of nocturnal awakenings, and subjective sleep quality. In addition, Arnedt et al ${ }^{18}$ concluded that online CBT-I is just as effective as therapist delivered treatment, and Sato et al ${ }^{19}$ outlined that online CBT-I also ameliorates insomnia symptoms in those individuals who have unsuccessfully undergone pharmacotherapy. Moreover, a report exploring the digital attitudes and behaviours of adults aged $70+$ years pointed out that $64 \%$ search for information online "a lot of the time", and $20 \%$ "all the time", suggesting that older adults are digitally literate. $^{20}$

\section{Aims}

This pilot study was conducted to investigate the feasibility of a non-randomised trial to examine the impact of online CBT-I on complaining compared with non-complaining sleepers aged 60-80 years in Western Australia. Participants were invited to examine an online CBT-I programme for user friendliness and programme content to ensure that this programme will be appropriate for a subsequent trial (aim 1). In addition, participants were asked to provide feedback on whether they considered the online CBT-I programme to be suitable for non-complaining poor sleepers (aim 2). Non-complaining poor sleepers are unlikely to participate in research or report to sleep clinics, however, it can be hypothesised that as they have suboptimal sleep, they too would benefit from a sleep education programme. Furthermore, it was important to evaluate digital literacy, i.e. to ascertain older adults' use of the internet, social media and other online resources, and any barriers to participation they considered relevant (aim 3). Finally, we planned to analyse qualitative participant feedback about the acceptability of the programme (aim 4).

\section{Methods}

\section{Design}

A single-arm design with an embedded qualitative component was utilised in this study. Full ethics approval was received from the Edith Cowan University Ethics committee under reference 2019-00449KUTZER. As the feasibility study involved piloting a component of the trial, the CONSORT extension to pilot and feasibility trials ${ }^{21}$ was implemented as per the guidelines for reporting non-randomised pilot and feasibility studies; 22 the CONSORT check list can be seen in Appendix I. 
The feasibility study was conducted in two phases - phase 1 involved recruiting a consumer via the University Consumer and Community Involvement Programme to review draft study documents for the proposed non-randomized trial and to provide feedback about the study design, including materials, and the appropriateness of the language used. The consumer only expressed concerns about the trial being conducted exclusively online, querying whether that would be the best format for the target group. They suggested that a paper copy of the questionnaire could be provided or that the study participant should be able to choose between an electronic or a paper-based study format. Taking this feedback into account it was decided to provide paper copies for the participant information sheet and the consent forms in addition to the electronic format, in case participants found it difficult to scan, sign and return their study documentation. However, we also made the decision to continue providing the questionnaire in its current online format. The rationale here was that the study aim was to test an intervention provided exclusively online, and that it could therefore be assumed that a potential participant would require a certain level of familiarity with navigating the internet and online resources to use the CBT-I portal. We did however include an additional question in our online survey enquiring about the frequency with which study participants used the internet to access health information to assess their level of familiarity with engaging in health topics online. In addition, whilst we wanted to acknowledge possible technological barriers, we also wanted to be aware of any potential age-based stereotypes such as the suggestion that all older individuals struggle to use new media.

Accessibility and suitability of the study design were further tested in phase 2 of the feasibility study with a specific focus on evaluating an online, unguided CBT-I programme. Participants were required to sign up to a free online platform to complete the first one out of four online CBT-I modules plus accompanying homework. In addition, users were asked to provide feedback via an online post-intervention questionnaire. The questionnaire collated information about the participant's age, gender and insomnia status (namely no insomnia present or insomnia present, and if so, presence of chronic insomnia), how the user rated the programme's content and general experience, ease of use, its accessibility (e.g. did they experience any problems logging on), their favourite and least favourite elements of the programme, how useful they considered the programme to be for a person with insomnia and would they recommend it to a friend or family member (also the reason for recommending or not recommending the programme), as well as frequency of accessing health information online. In addition, participants were asked if they deemed the planned study design suitable for use with non-complaining poor sleepers.

\section{Description of intervention}

The intervention consisted of an online, self-help, unguided CBT-I programme (This Way Up Managing Insomnia $\left.{ }^{\circledR}\right) .{ }^{23}$ This Way Up is on online platform accessible via the This Way Up website, ${ }^{24}$ which offers self-help and clinician guided courses for mental health, chronic illness, and general well-being.

The Managing Insomnia programme is comprised of four modules in an illustration-based format and can be completed on a computer or mobile phone. Lesson one educates the user about insomnia and sleep hygiene, lesson two addresses sleep interfering thoughts and behaviours, lesson three introduces 
bedtime restriction therapy (a therapeutic approach endorsing temporary adherence to a restricted sleep wake schedule), and lesson four focusses on relaxation techniques and relapse prevention. The programme provides broad topic coverage of the most common factors present in insomnia (e.g. difficulty falling asleep, difficulty staying asleep, and waking too early) ${ }^{25}$

Each of the four lessons takes approximately twenty minutes to complete, with additional compulsory homework, which summarises the session content and includes practical exercises to further consolidate learning. Reminders are emailed or sent by text message automatically once a new session becomes available, and there is also an option to set an online appointment for the next course module. In addition to the course unit and homework, participants are required to complete a sleep diary, which can be downloaded from the website or filled in online. At the start of each new module participants fill in selfreport questionnaires about their general mood and well-being, such as the Kessler 10-item Psychological Distress Scale (K-10), ${ }^{26}$ a measure of psychological distress, the Insomnia Severity Index (ISI), ${ }^{27}$ a measure of sleep satisfaction, and the World Health Organization 5-item Well-Being Index (WHO-5), ${ }^{28,29}$ a measure of general wellbeing, which is completed before lessons 1 and 4 .

Participants in this feasibility study were asked to complete Lesson 1 only - The Science of Sleep and Understanding Your Insomnia - and were instructed to select the self-help version of the programme. Participants were not obliged to complete any other module of the programme.

\section{Participants and setting}

Participants were recruited using convenience sampling, utilising professional and personal networks, or asked to complete the feasibility trial if they were screened out of another study due to obstructive sleep apnoea. There were no inclusion criteria other than being aged 60-80 years. Recruitment took place between October 2019 and October 2020. Participants were approached via email and asked if they were interested in taking part, and if they expressed interest were emailed a study overview and an information $\&$ consent form. They were required to carefully read, sign and return the form. Participants also had the option to receive the information \& consent form via mail and return it using a pre-paid self-addressed envelope. Since there was no direct contact with participants, recruitment and enrolment was not impacted by the outbreak of the COVID-19 pandemic.

\section{Procedure}

After returning their signed consent form, participants were emailed the study instructions. All participants received the same programme instructions, which included information on how to access the online programme, create an account, how to navigate the online platform and how to complete lesson 1 and review homework and additional resources. The instruction email further contained a link to the brief post-intervention questionnaire. Once participants confirmed that they had completed lesson 1 and the questionnaire, they were mailed a voucher to reimburse them for the time commitment they had made. The recompense was in line with Edith Cowan University guidelines governing the reimbursement of consumers. The participant flow chart is outlined in Fig. 1. 


\section{Data analysis}

Acceptability, utility and accessibility of the intervention were assessed as follows: The intervention was considered acceptable, user-friendly, and accessible if $75 \%$ or more of the participants rated the online CBT-I module positively (information Very easy- or Fairly easy to understand, Definitely useful or fairly useful, and if they did not have any problems with accessing the programme). Answers to open-ended questions on the questionnaire were analysed using content analysis. Content analysis uses distinct categories which are defined prior to the analysis of the research data and emphasises the contextual meaning of the text. Subsequently, coding and theme development was guided by existing concepts and aimed to identify manifest themes that showed the participants' assessment of the online CBT-I programme with specific focus on acceptability, utility, and accessibility. Here, text data was obtained from the open-ended survey questions contained in the post-intervention questionnaire. ${ }^{30}$

We initially aimed to enrol 25 participants for the feasibility study, since preliminary data analyses and an a priori power analysis indicated that we would need to recruit a minimum of 23 participants to detect a correlation coefficient of 0.5 or greater. However, due to time as well as financial constraints, we had to limit recruitment to 12 participants. The analysis of quantitative outcomes was therefore primarily completed using descriptive statistics.

\section{Results}

To date, 12 participants have completed the feasibility study. A total of 22 individuals were approached and 12 participants completed the online lesson and questionnaire (phase 2 of the feasibility study). This included six females and five males, with one participant not indicating which gender they identified with. Participants were aged between 61 and 74 years (mean $=67.4, S D=3.8$ ). Less than half of the participants ( $n=5$ participants, $45.5 \%$ ) reported having insomnia, and of those $80 \%$ ( $n=4$ participants) advised having chronic insomnia.

Results Aim 1: Acceptability, utility and accessibility of the intervention. Acceptability. when asked how useful participants perceived the programme to be, 11 participants $(91.7 \%)$ stated that they found the lesson Definitely useful $(n=7,58.3 \%)$ or Fairly useful $(n=4,33.3 \%)$. When asked if they would recommend the programme to a friend or family member with insomnia, 11 out of 12 users said they would (91.7\%). Utility. 11 out of $12(91.7 \%)$ participants found the programme Very easy $(n=7$ participants, $58.3 \%$ ) or Fairly easy ( $\mathrm{n}=4$ participants, 33.3\%) to understand, with one user reporting that the programme was Fairly difficult to understand (8.3\%). Accessibility. 11 out of 12 users said they did not have any problems accessing the lesson (91.7\%) and one had had problems (8.3\%). The user with access problems reported a possible website glitch, meaning they were re-directed to a different page when attempting to access the online lesson. 
Results Aim 2: Hypothetical scenario. Respondents were asked a hypothetical question (Imagine a researcher asks you to wear a sleep watch that measures how well you sleep. After you have worn the watch for a few days, the researcher tells you do not sleep as well as you thought, even though you have never considered yourself to be a person who has insomnia. Based on this scenario, would you be more likely or less likely to complete the online CBT-I programme This Way Up - Managing Insomnia ${ }^{\circledR}$ ?). Eleven participants (91.7\%) stated that they would be more likely to complete the online CBT-I programme $(\mathrm{n}=7$ probably more likely, $\mathrm{n}=4$ definitely more likely).

Results Aim 3: Older adults' internet use. Four individuals used the internet weekly to access health and medical information (36.4\%), two accessed information on a monthly basis (18.2\%), three less than monthly (27.3\%), and two had never accessed the internet for health information (18.2\%). None of the 12 participants retrieved online health information daily. Males were more likely to access health information on a weekly basis than females (three males versus one female). Those with insomnia accessed online health information just as frequently as those without sleep problems.

Findings Aim 4: Qualitative feedback. The following section summarises the findings of the content analysis of open text data. Acceptability. Participants expressed generally positive feedback about the content of the online intervention. They described the content as helpful, informative, and easy to implement:

"I thought it was very informative and highlighted problems associated with insomnia" (P10, F, chronic insomnia)

"Useful and easy to implement the recommended solution" (P3, M, insomnia)

"It is certainly helpful" (P11, M, no insomnia)

They also appeared receptive of the hypothetical non-complaining poor sleeper scenario, expressing their willingness to take part in the programme:

"Because I need it even more than I realised" (P7, F, insomnia status unknown)

As the main reasons for being open to participating in the online sleep programme, study participants cited their desire to learn more about their sleep, as well as acknowledging the importance of good sleep for their health:

"It whetted my appetite to try to overcome insomnia (P2, F, chronic insomnia)

"Looking for a way towards a better sleeping pattern without medication" (P9, gender not known, chronic insomnia)

"Since healthy sleep pattern is necessary for overall good health" (P12, F, no insomnia)

They also noted that the programme had a scientific, evidence-based foundation: 
"Very informative and delivers clinical/scientific information in an (sic) logical and easy to understand manner" (P3, M, insomnia)

"If the evidence is there, then why not follow it" (P4, M, no insomnia)

Participants did express concerns about the fact that programme could not be individually tailored, e.g. to the level of insomnia experienced: "For people with (sic) mild to moderate insomnia it's probably very helpful" (P8, F, chronic insomnia), to account for co-morbid conditions, such as anxiety: "[my partner had] severe insomnia due to Generalised Anxiety Disorder." (P5, M, no insomnia), nor did it provide advice for shift workers on how to manage insomnia: "Very difficult to have set sleep times when a shift worker. Lots of shift workers having sleeping problems." (P1, F, no insomnia), that strategies could not be tailored to address individual difficulties with sleep, e.g. sleep onset insomnia: "Most of the information doesn't seem to apply to me, but I will try two of the strategies and see if it makes a difference" (P8, F, chronic insomnia), the fact that the programme was unguided online therapy without human interaction: "I do think the course is great but I do have some concerns about not talking with someone face to face. I think people can easily gloss over issues in the online course instead of delving deeper" (P7, F, insomnia status unknown), the perceived length of the programme: "Short" (P6, $M$, no insomnia), and that the programme content was too simplistic: "I thought is (sic) was childish and simplistic" (P5, M, no insomnia).

Participants further mentioned a lack of visual engagement in the design components of the programme:

"Little engagement, graphic could be jazzed up, no video component" (P3, M, insomnia)

"The illustrations were poor" (P10, F, chronic insomnia)

\section{Utility}

Participants unanimously described the programme as easy to use and clearly explained

"It's easy to read and straight forward information" (P2, F, chronic insomnia)

"Easy to follow and some great suggestions" (P1, F, no insomnia)

\section{Accessibility}

The majority of participants stated that they did not have problems accessing the programme. However, two participants mentioned difficulty with accessing the online platform ("I couldn't get into it I kept getting the K10. It took 4 or 5 attempts to get in. Finally I did." P8, F, chronic insomnia), or confusion over which access instructions to follow - the programme instructions or the additional access instructions that were provided by the researchers ("I initially found it confusing as to which instructions I was to follow, yours or "This Way up". Once that was established all was fine" P11, M, no insomnia).

The content of the insomnia programme elicited positive responses in both participants with and without insomnia. However, only participants with insomnia reiterated a desire to learn more about sleep, even 
though both those with and without sleep difficulties acknowledged the importance of good sleep for overall health and the importance of having a scientific, evidence-based foundation for the programme.

It is with this prior knowledge in place that participants without insomnia may have evaluated the online CBT-I programme from a hypothetical point of view. One participant, who learned about insomnia vicariously, deemed the programme useful for those who did not have a lot of prior knowledge about insomnia, but evaluated the programme content and delivery from their partner's perspective:

"[They] would have found this module very irritating in its glib and simplistic solutions to what was a major factor in [their] loss of quality of life. I can say with great certainty [they] would not have continued with the course, because it has an air of 'look, these are the tried-and-true solutions, if they aren't working for you then it's your fault'" (P5, M, no insomnia)

\section{Discussion}

The outcomes of this feasibility study suggest that study participants viewed the online CBT-I programme as acceptable, useful and accessible, independent of their insomnia status. All participants had some prior knowledge of the health aspects of sleep; in addition, the online module appeared to elicit a desire to learn more about insomnia in those participants with sleep problems. Qualitative analysis also produced some more in-depth knowledge about issues that were seen as barriers by the participants. Such barriers were that the programme was not interactive and could not be tailored to individual circumstances, was too simplistic, and the fact that the programme was lacking human interaction and was not very visually engaging.

User complaints relating to oversimplified content appear to be a common occurrence with online CBT interventions, with even those providing more interactive content and using various forms of media reporting such feedback. ${ }^{31}$ Nonetheless, we considered broad coverage of the topic of insomnia, using accessible language and an uncomplicated media format essential for reaching participants from all socioeconomic backgrounds, those with English as a second language and those feeling less at ease with using online platforms. Complaints of over-simplified content or lack of human interaction, however, do not appear to alter the efficacy of digitally delivered CBT-I programmes, with one systematic review highlighting that online CBT-I was as successful at improving insomnia severity, wake after sleep onset, sleep onset latency and other outcome variables as a face-to-face intervention, ${ }^{17}$ and another suggesting that the role of therapeutic alliance in CBT-I might not be as crucial as previously suggested. ${ }^{32}$ What is more, whilst web-based interventions are often prone to low levels of user adherence, ${ }^{33,34}$ older adults, in contrast, have been shown to demonstrate high levels of adherence to online interventions. ${ }^{35,36}$ This makes internet-based CBT-I a viable method of treatment delivery for older adults, particularly in remote areas, given the scarcity of trained clinicians able to provide in-person CBT-I. ${ }^{37}$

Limitations of this study were that it was underpowered, and the fact that individuals who are not as familiar with internet use and social media or who have only limited internet access may have been less 
likely to take part in this research. However, our analysis showed that there was sufficient interest and knowledge of online resources amongst the 60-80 age group in Western Australia. In addition, participants only completed one module out of a total of four for this trial, which may have given them limited insight into the programme. Participants further received a reimbursement for their time investment, which may have skewed their perception of the programme. Finally, although study recruitment took place during the onset of the COVID-19 pandemic, we did not consider the impact this might have had on attrition or completion rates. Still, the lasting nature of the pandemic has further emphasised the importance of making digitally delivered health care intervention available to the general population and at-risk groups, such as older individuals. What is more, our feasibility study only examined the intention to adhere to the programme but did not assess actual attrition rates. Examination of adherence in a naturalistic setting, as e.g. conducted by Grierson et al, ${ }^{25}$ might be advisable for future research.

\section{Conclusion}

In summary, the feedback provided in this feasibility trial providing a self-guided online CBT-I intervention was positive amongst 60-80-year-olds in Western Australia. Participants were receptive to undertaking an online intervention for insomnia. They reported that the programme was easy to understand, with some minor comments addressing the fact that the programme cannot be tailored to meet individual needs. The programme appears to be suitable overall for this age group.

\section{Declarations}

\section{Ethics approval and consent to participate}

This study has received approval from the Edith Cowan University Ethics committee under reference 2019-00449-KUTZER. All participants provided written consent.

\section{Consent for publication}

Not applicable.

\section{Availability of data and materials}

The data that support the findings of this study are available on the figshare repository on $10.6084 / \mathrm{m}$ 9.figshare. 14572092 but restrictions apply to the availability of these data, which were used under license for the current study, and so are not publicly available. Data are however available from the authors upon reasonable request.

\section{Competing interests}

The authors declare that they have no competing interests. 


\section{Funding}

YK received an Edith Cowan University, School of Medical and Health Sciences School Collaboration Grant. Partial funds from the Edith Cowan University, School of Medical and Health Sciences School Collaboration Grant were used to obtain statistical advice.

\section{Authors' contributions}

YK designed the study, collected and analysed the data and wrote the initial manuscript draft. MS provided input regarding the qualitative data analysis, and EQ and LW contributed to the study design. All authors made substantial contributions to revising the initial manuscript and read and approved the final manuscript.

\section{Acknowledgements}

We wish to thank our study participants for their support, and Shih Ching Fu for his advice with regard to the data collection for this project. YK is supported through an Australian Government Research Training Program Scholarship

\section{Authors' information}

This feasibility trial contributes to the Doctor of Philosophy at Edith Cowan University for YK.

\section{References}

1. Ancoli-Israel S. Sleep and aging: prevalence of disturbed sleep and treatment considerations in older adults. J Clin Psychiatry 2005;66 Suppl 9:24-30.

2. Foley DJ, Monjan AA, Brown SL, Simonsick EM, Wallace RB, Blazer DG. Sleep complaints among elderly persons: an epidemiologic study of three communities. Sleep 1995;18(6):425-32.

3. Sateia MJ. International classification of sleep disorders-third edition: highlights and modifications. Chest 2014;146(5):1387-94.

4. Lichstein KL. Insomnia identity. Behav Res Ther 2017;97:230-41.

5. Castelnovo A, Ferri R, Punjabi NM, Castronovo V, Garbazza C, Zucconi M, et al. The paradox of paradoxical insomnia: A theoretical review towards a unifying evidence-based definition. Sleep Med Rev 2019;44:70-82.

6. Carney CE, Edinger JD, Morin CM, Manber R, Rybarczyk B, Stepanski EJ, et al. Examining maladaptive beliefs about sleep across insomnia patient groups. J Psychosom Res 2010;68(1):57-65.

7. Chang S, Ma Q, Seow E, Lau YW, Satghare P, Hombali A, et al. Sleep beliefs and attitudes and the association with insomnia among psychiatric outpatients. J Ment Health 2020;29(1):33-9. 
8. Eidelman P, Talbot L, Ivers H, Bélanger L, Morin CM, Harvey AG. Change in dysfunctional beliefs about sleep in behavior therapy, cognitive therapy, and cognitive-behavioral therapy for insomnia. Behav Ther 2016;47(1):102-15.

9. Lancee J, Effting M, van der Zweerde T, van Daal L, van Straten A, Kamphuis JH. Cognitive processes mediate the effects of insomnia treatment: evidence from a randomized wait-list controlled trial. Sleep Med 2019;54:86-93.

10. Lancee J, Eisma MC, van Straten A, Kamphuis JH. Sleep-related safety behaviors and dysfunctional beliefs mediate the efficacy of online CBT for insomnia: A randomized controlled trial. Cogn Behav Ther 2015;44(5):406-22.

11. Sánchez-Ortuño MM, Edinger JD. A penny for your thoughts: patterns of sleep-related beliefs, insomnia symptoms and treatment outcome. Behav Res Ther 2010;48(2):125-33.

12. Schwartz DR, Carney CE. Mediators of cognitive-behavioral therapy for insomnia: a review of randomized controlled trials and secondary analysis studies. Clin Psychol Rev 2012;32(7):664-75.

13. Sella E, Cellini N, Miola L, Sarlo M, Borella E. The Influence of Metacognitive Beliefs on Sleeping Difficulties in Older Adults. Appl Psychol Health Well Being 2019;11(1):20-41.

14. Ree MJ, Junge M, Cunnington D. Australasian Sleep Association position statement regarding the use of psychological/behavioral treatments in the management of insomnia in adults. Sleep Med 2017;36:S43-S7.

15. Lorenz N, Heim E, Roetger A, Birrer E, Maercker A. Randomized Controlled Trial to Test the Efficacy of an Unguided Online Intervention with Automated Feedback for the Treatment of Insomnia. Behav Cogn Psychother 2019;47(3):287-302.

16. Rybarczyk B, Lund HG, Garroway AM, Mack L. Cognitive behavioral therapy for insomnia in older adults: Background, evidence, and overview of treatment protocol. Clin Gerontol 2013;36(1):70-93.

17. Zachariae R, Lyby MS, Ritterband LM, O'Toole MS, O'Toole MS. Efficacy of internet-delivered cognitivebehavioral therapy for insomnia - A systematic review and meta-analysis of randomized controlled trials. Sleep Med Rev 2016;30:1-10.

18. Arnedt JT, Conroy DA, Mooney A, Furgal A, Sen A, Eisenberg D. Telemedicine versus face-to-face delivery of cognitive behavioral therapy for insomnia: a randomized controlled noninferiority trial. Sleep 2021;44(1).

19. Sato D, Yoshinaga N, Nagai E, Nagai K, Shimizu E. Effectiveness of Internet-Delivered Computerized Cognitive Behavioral Therapy for Patients With Insomnia Who Remain Symptomatic Following Pharmacotherapy: Randomized Controlled Exploratory Trial. J Med Internet Res 2019;21(4):e12686. 
20. Care Connect. The Connectivity Report [Internet]. 2018 [cited 202103 March]; Available from: https://www.careconnect.org.au/wp-content/uploads/2018/04/Care-Connect-Connectivity-Report-April2018-Full-report.pdf

21. Eldridge SM, Chan CL, Campbell MJ, Bond CM, Hopewell S, Thabane L, et al. CONSORT 2010 statement: extension to randomised pilot and feasibility trials. 2016;355:i5239.

22. Lancaster GA, Thabane L. Guidelines for reporting non-randomised pilot and feasibility studies. Pilot and Feasibility Studies 2019;5(1):114.

23. This Way Up. This Way Up - Managing Insomnia ${ }^{\circledR}[$ Internet]. [date unknown] [cited 2021 Mar 03]; Available from: https://thiswayup.org.au/courses/managing-insomnia-course/

24. This Way Up. Learn practical tools to take care of your mental health [Internet]. [date unknown] [cited 2021 Mar 03]; Available from: https://thiswayup.org.au/

25. Grierson AB, Hobbs MJ, Mason EC. Self-guided online cognitive behavioural therapy for insomnia: A naturalistic evaluation in patients with potential psychiatric comorbidities. J Affect Disord 2020;266:30510 .

26. Kessler RC, Andrews G, Colpe LJ, Hiripi E, Mroczek DK, Normand SLT, et al. Short screening scales to monitor population prevalences and trends in non-specific psychological distress. Psychol Med 2002;32(6):959-76.

27. Bastien $\mathrm{CH}$, Vallières $\mathrm{A}$, Morin $\mathrm{CM}$. Validation of the Insomnia Severity Index as an outcome measure for insomnia research. Sleep Med 2001;2(4):297-307.

28. Topp CW, Østergaard SD, Søndergaard S, Bech P. The WHO-5 Well-Being Index: A systematic review of the literature. Psychother Psychosom 2015;84(3):167-76.

29. Bech P, Gudex C, Staehr Johansen K. The WHO (Ten) Well-Being Index: Validation in diabetes. Psychother Psychosom 1996;65(4):183-90.

30. Hsieh H-F, Shannon SE. Three Approaches to Qualitative Content Analysis. Qual Health Res 2005;15(9):1277-88.

31. Sweeney L, Moss-Morris R, Czuber-Dochan W, Norton C. Pain management in inflammatory bowel disease: feasibility of an online therapist-supported CBT-based self-management intervention. Pilot and Feasibility Studies 2021;7(1):95.

32. Carlbring P, Andersson G, Cuijpers P, Riper H, Hedman-Lagerlöf E. Internet-based vs. face-to-face cognitive behavior therapy for psychiatric and somatic disorders: an updated systematic review and meta-analysis. Cogn Behav Ther 2018;47(1):1-18. 
33. Ryan C, Bergin M, Wells JSG. Theoretical Perspectives of Adherence to Web-Based Interventions: a Scoping Review. Int J Behav Med 2018;25(1):17-29.

34. Wangberg SC, Bergmo TS, Johnsen J-AK. Adherence in Internet-based interventions. Patient preference and adherence 2008;2:57-65.

35. Kelders SM, Van Gemert-Pijnen JEWC, Werkman A, Nijland N, Seydel ER. Effectiveness of a Webbased Intervention Aimed at Healthy Dietary and Physical Activity Behavior: A Randomized Controlled Trial About Users and Usage. 2011;13(2):e32.

36. Rothert K, Strecher VJ, Doyle LA, Caplan WM, Joyce JS, Jimison HB, et al. Web-based Weight Management Programs in an Integrated Health Care Setting: A Randomized, Controlled Trial. 2006;14(2):266-72.

37. Brooks AT, Tuason RT, Chakravorty S, Raju S, Ritterband LM, Thorndike FP, et al. Online cognitive behavioral therapy for insomnia (CBT-I) for the treatment of insomnia among individuals with alcohol use disorder: study protocol for a randomized controlled trial. Pilot and Feasibility Studies 2018;4(1):183.

\section{Figures}




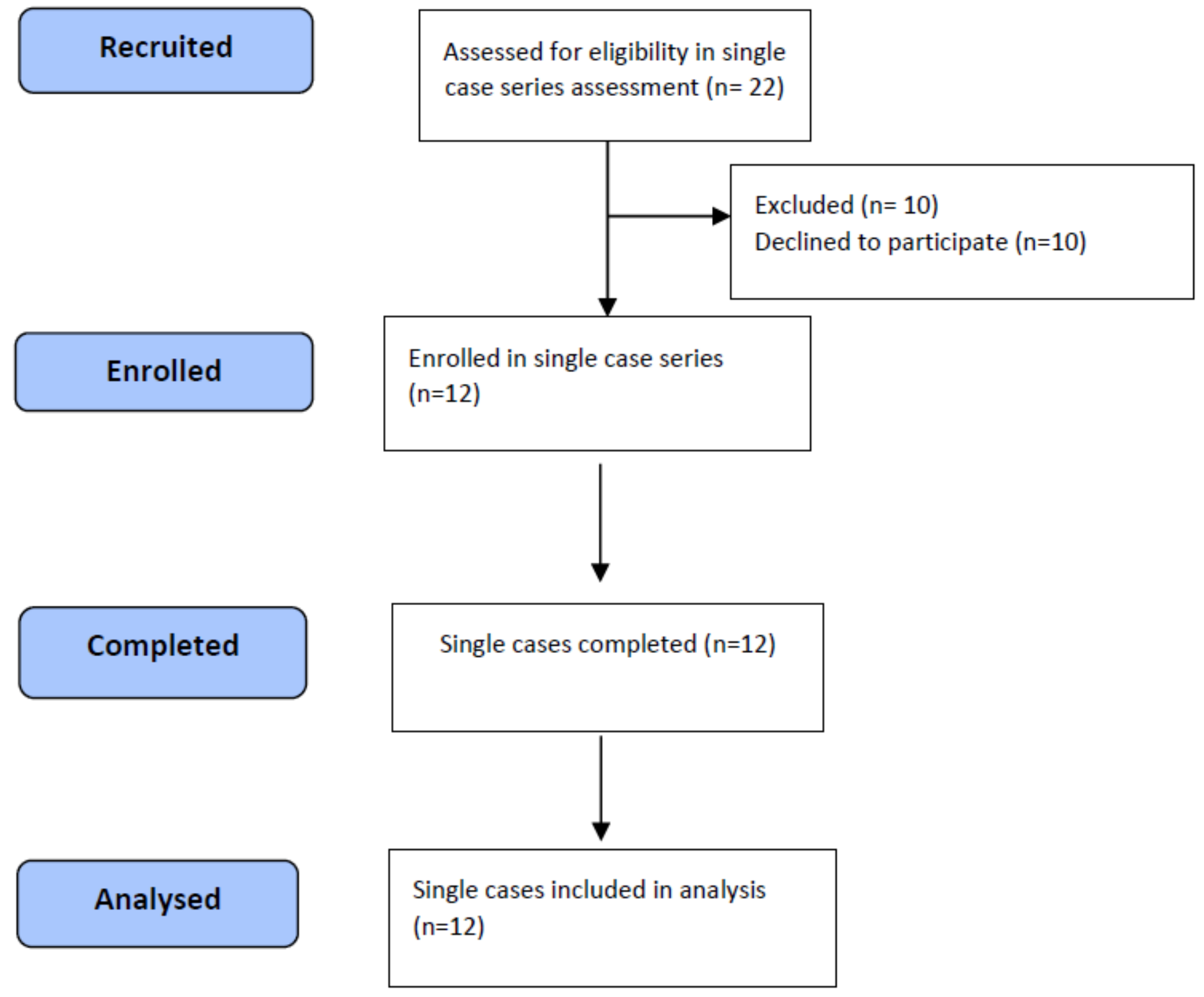

Figure 1

Participant flow chart

\section{Supplementary Files}

This is a list of supplementary files associated with this preprint. Click to download.

- 210609CONSORTextension.docx 